\title{
Erratum: Configurational temperature in dusty plasmas [Phys. Rev. E 99, 063203 (2019)]
}

\author{
Michael Himpel and André Melzer
}

(Received 30 January 2020; accepted 3 February 2020; published 18 February 2020)

DOI: 10.1103/PhysRevE.101.029902

In the original paper, we encountered a mistake in the presented formulas and accordingly in the software implementation of these formulas. The impact of this mistake depends on some parameters. The analysis of simulated data is nearly unchanged. The analysis of real measurements is corrected towards higher temperatures, which leads to lower estimations of charges.

The necessary corrections are as follows:

(1) Equation (6) in the original paper must be replaced by

$$
\mathbf{F}_{i j}^{\mathrm{el}}=\frac{Q_{i} Q_{j}}{4 \pi \epsilon_{0}}\left(\frac{1}{r_{i j}^{2}}+\frac{1}{r_{i j} \lambda_{\mathrm{s}}}\right) e^{-r_{i j} / \lambda_{\mathrm{s}}} \frac{\left(\mathbf{q}_{i}-\mathbf{q}_{j}\right)}{r_{i j}} .
$$

(2) Equation (7) in the original paper must be replaced by

$$
\mathbf{F}_{j}^{\mathrm{el}}=\sum_{i \neq j}^{N} \frac{Q_{i} Q_{j}}{4 \pi \epsilon_{0}}\left(\frac{1}{r_{i j}^{2}}+\frac{1}{r_{i j} \lambda_{\mathrm{s}}}\right) e^{-r_{i j} / \lambda_{\mathrm{s}}} \frac{\left(\mathbf{q}_{i}-\mathbf{q}_{j}\right)}{r_{i j}} .
$$

(3) Equation (8) in the original paper must be replaced by

$$
\nabla_{j} \cdot \mathbf{F}_{j}^{\mathrm{el}}=\sum_{i \neq j}\left[\frac{Q_{i} Q_{j}}{4 \pi \epsilon_{0}}\left(\frac{1}{r_{i j}^{3}}+\frac{1}{r_{i j}^{2} \lambda_{\mathrm{s}}}+\frac{1}{r_{i j} \lambda_{\mathrm{s}}^{2}}\right) e^{-r_{i j} / \lambda_{\mathrm{s}}}\right] .
$$

(4) Figure 3 in the original paper must be replaced by Fig. 3. The changes are small, and no changes in the text are necessary.

(5) Figure 7 in the original paper must be replaced by Fig. 7. The corresponding text from Sec. IV C: "Approximating again the screening length by the interparticle distance,

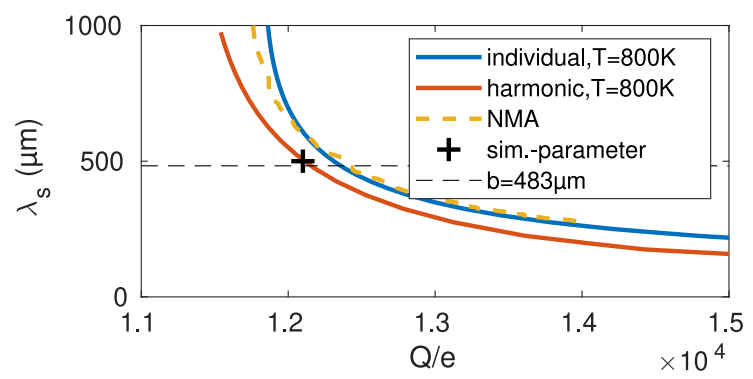

FIG. 3. The plot shows curves on the $Q-\lambda_{\mathrm{s}}$ plane that yield the parameters of the simulated dust cluster. The dashed line represents the results from the mode analysis, the two solid lines are obtained by setting $T_{\text {conf }}^{\text {ind }}(Q, \lambda)=800 \mathrm{~K}$ and $T_{\text {conf }}^{\mathrm{c}}(Q, \lambda)=800 \mathrm{~K}$. The crossmark is placed at the actual simulation parameter position $\left(\lambda_{\mathrm{s}}=500 \mu \mathrm{m}\right.$ and $Q=12100 e$ ). The dashed horizontal line indicates the mean interparticle distance.

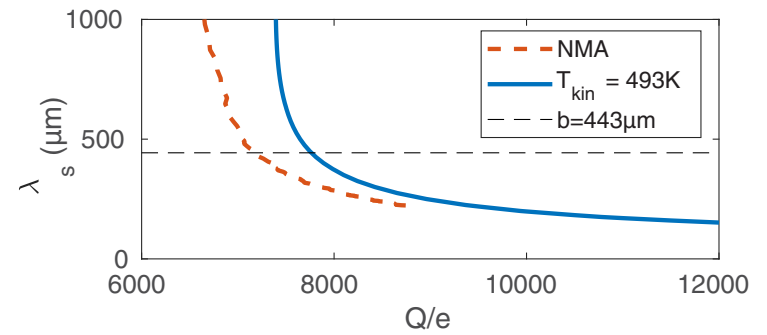

FIG. 7. $\lambda_{\mathrm{s}}(Q)$ functions for a measured dust cluster at $p=$ 8.2 Pa. The solid curve is retrieved by intersecting the configurational temperature surface at the measured kinetic temperature of $493 \mathrm{~K}$. The thick dashed line is obtained from the mode analysis of the cluster. The thin dashed line indicates the measured mean interparticle distance.

i.e., $\lambda_{s} \approx b=443 \mu \mathrm{m}$, the particle charge can be estimated as $7200 e$ for the NMA analysis and $9600 e$ for the configurational temperature analysis." has to be changed to: "Approximating again the screening length by the interparticle distance, i.e., $\lambda_{s} \approx b=443 \mu \mathrm{m}$, the particle charge can be estimated as $7200 e$ for the NMA analysis and 7900e for the configurational temperature analysis."

(6) Figure 8 in the original paper has to be replaced by Fig. 8.

(7) Figure 9 in the original paper has to be replaced by Fig. 9. The dust charges retrieved in this corrected version are somewhat smaller than in the original paper. A change in the text is, however, not necessary.

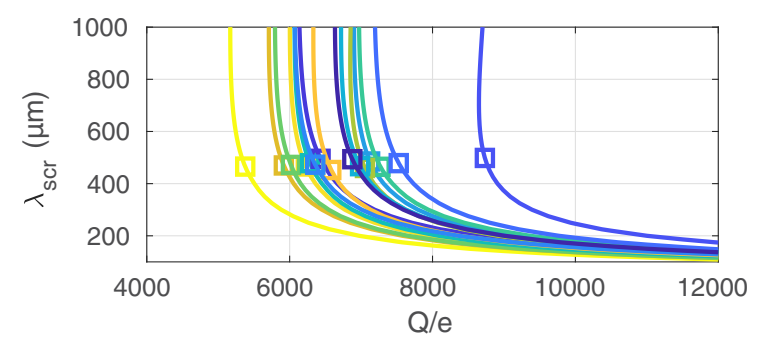

FIG. 8. The different $\lambda_{\mathrm{s}}(Q)$ curves are obtained from a dust cluster under different neutral gas pressures. The pressure is color coded and increases from blue to yellow color just as in Fig. 9. The function values using the nearest-neighbor distances between the particles as a measure for $\lambda_{\mathrm{s}}$ are highlighted by squares. 


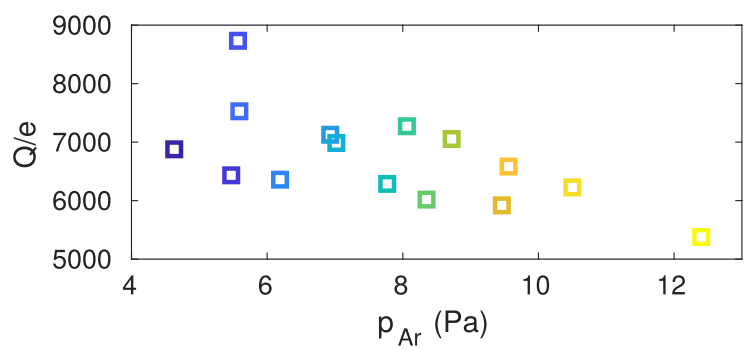

FIG. 9. The particle charge dependence on the neutral gas pressure. The colors of the markers reflect the pressure accordingly.

(8) Figure 12 in the original paper has to be replaced by Fig. 12. The text in Sec. IV E, third paragraph, "The particle charge is found to be about $Q \approx 7000 \pm 1000 e$ at a screening length $\lambda_{\mathrm{s}}$ that we again have estimated to be close to the interparticle distance $b=350 \mu \mathrm{m}$." has to be changed to "The particle charge is found to be about $Q \approx 6500 \pm 1000 e$ at a screening length $\lambda_{\mathrm{s}}$ that we again have estimated to be close to the interparticle distance $b=350 \mu \mathrm{m}$."

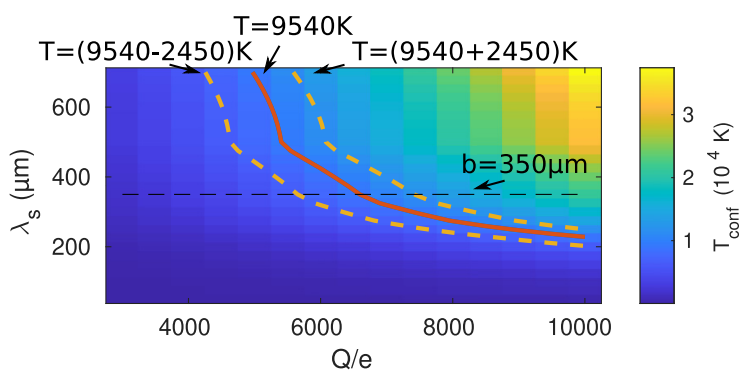

FIG. 12. Configurational temperature of a volumetric measurement in a dust cloud. The solid line indicates the $\lambda_{\mathrm{s}}(Q)$ function at a given temperature of $9540 \pm 2450 \mathrm{~K}$.

The corrected version of this paper including all modifications is available in Ref. [1].

We want to thank F. Wieben for the fruitful discussions that resulted in the discovery of the mistake in the formulas.

[1] M. Himpel and A. Melzer, arXiv:2001.09741. 